\title{
Teoria fenomenológica do processo de relaxação dielétrica
}

(Phenomenological theory of dielectric relaxation process)

\author{
Renê Robert 1 \\ Departamento de Engenharia Elétrica, UFPR, Curitiba, PR, Brasil \\ Recebido em 18/05/04; Aceito em 19/07/04
}

\begin{abstract}
O objetivo deste trabalho é introduzir o conceito de não simultaneidade entre causa e efeito, o que não é muito comum em diversos problemas de física e biologia, mostrando como tratá-los e verificando em que condições os dois modelos conduzem a resultados similares. Para este estudo usaremos os dielétricos. A teoria de Pellat-Debye para a relaxação dielétrica é modificada introduzindo-se uma atraso de resposta (polarização) à ação de um estímulo (campo elétrico), de modo a tornar válido o princípio de causalidade.
\end{abstract}

Palavras-chave: relaxação dielétrica, simultaneidade.

The goal of this work is to introduce the concept of non simultaneity between cause-effect, which is not very common in several problems of physics and biology, showing how to treat these problems and verifying the results. For this study we will use the dielectrics. Pellat-Debye's theory for dielectric relaxation is modified introducing a delay in the Response ( polarization ) to the action (electric field), so as the validity of the causality principle is satisfied.

Keywords: dielectric relaxation, simultaneity.

\section{Introdução}

H. Pellat (1899) [1] provou experimentalmente que os dielétricos manifestam uma polarização inicial instantânea à qual é acrescentada uma segunda polarização variável no tempo, sendo que esta última tende a um valor de equilíbrio com uma velocidade proporcional à diferença entre o valor de equilíbrio e o valor presente. Analiticamente pode-se escrever $P(t)=P_{1}(t)+P_{2}(t)$, onde $P_{1}(t)$ é o valor da polarização instantânea e $P_{2}(t)$ é o valor da polarização variável no tempo. Esta última obedece a equação diferencial [1,2,3]: $\tau \frac{d P_{2}(t)}{d t}=P_{s}-P_{2}(t)$, onde $P_{s}$ é a polarização de equilíbrio e $\tau$ é chamado tempo de relaxação. Define-se relaxação como o atraso da resposta de um sistema devido a variação das forças sob o qual esta sujeito.

Este modelo permite calcular a permissividade relativa do dielétrico, que no caso de campo alternado senoidal com frequiência angular $\omega$, dá origem a uma função complexa $\tilde{\varepsilon}(\omega)=\varepsilon^{\prime}(\omega)-i \varepsilon^{\prime \prime}(\omega)$. As expressões de $\varepsilon^{\prime}(\omega)$ e $\varepsilon^{\prime \prime}(\omega)$ são conhecidas como permis- sividade relativa verdadeira e fator de perda, respectivamente.

Neste trabalho, para efeito de cálculo, usa-se a hipótese de que o dielétrico é homogêneo, isolante perfeito e está colocado entre os eletrodos de um capacitor de placas planas e paralelas e despreza-se os efeitos de borda.

A equação diferencial proposta por Pellat afirma que $P_{2}(t)$ define, no mesmo instante, a derivada $\frac{d P_{2}(t)}{d t}$, o que não é compatível com o princípio de causalidade. O objetivo deste trabalho é verificar o que ocorre com o comportamento da polarização variável no tempo e com a permissividade relativa complexa quando introduzimos um atraso no tempo ou "time lag" $r$ em $P_{2}(t)$, isto é, substituímos $t$ por $(t-r)$.

\section{Teoria}

Quando submetemos um material dielétrico à ação de um campo elétrico uniforme $E(t)$, o dielétrico se polariza. A polarização é composta de duas partes: $P_{1}(t)$ resultante de processos eletrônicos e atômicos

\footnotetext{
${ }^{1}$ Enviar correspondência para Renê Robert. E-mail: rene@lactec.org.br.
} 
e $P_{2}(t)$ resultante de processos de orientação dipolar. Para baixas frequiências $P_{1}(t)$ segue o campo elétrico com desprezível atraso, de modo que $P_{1}(t)=$ $\chi_{1} \varepsilon_{0} E(t)$, onde $\chi_{1}$ é a susceptibilidade relativa em altas frequiências, ao passo que com $P_{2}(t)$ ocorre uma defasagem com relação ao campo elétrico. Um modelo $[1,2,3]$ simples e comumente usado para a definição de $P_{2}(t)$ é aquele dado pela seguinte equação:

$$
\tau \frac{d P_{2}(t)}{d t}=\chi_{2} \varepsilon_{0} E(t)-P_{2}(t)
$$

onde $\chi_{2}$ é a susceptibilidade elétrica relativa em baixas freqüências, $\varepsilon_{0}$ é a permissividade do vácuo, e $\tau$ é chamado tempo de relaxação. A grandeza $P_{s}=$ $\chi_{2} \varepsilon_{0} E(t)$ é chamada polarização de saturação.

Se $E(t)=E_{0} u(t)$, onde $E_{0}$ é constante e $u(t)$ é a função degrau de Heaviside, e $P_{2}(0)=0$, a solução da equação diferencial (1) é:

$$
P_{2}(t)=\chi_{2} \varepsilon_{0}\left[1-\exp \left(-\frac{t}{\tau}\right)\right] E_{0}
$$

Portanto, a polarização total é dada pela equação:

$$
P(t)=P_{1}+P_{2}=\left[\chi_{1}+\chi_{2}\left(1-\exp \left(-\frac{t}{\tau}\right)\right)\right] \varepsilon_{0} E_{0} .
$$

De maneira similar, se $E(t)=E_{0} \exp (i \omega t)$, a polarização total fica:

$$
\begin{gathered}
P(t)=P_{1}+P_{2}= \\
d\left(\chi_{1}+\frac{\chi_{2}}{1+i \omega \tau}\right) \varepsilon_{0} E_{0} \exp (i \omega t) .
\end{gathered}
$$

A permissividade relativa complexa é calculada, obtendo-se:

$$
\tilde{\varepsilon}=\varepsilon^{\prime}-i \varepsilon^{\prime \prime}=\chi_{1}+\frac{\chi_{2}}{1+i \omega \tau}
$$

onde $\varepsilon^{\prime}=\varepsilon_{\infty}+\frac{\varepsilon_{s}-\varepsilon_{\infty}}{1+\omega^{2} \tau^{2}}$ e $\varepsilon^{\prime \prime}=\frac{\left(\varepsilon_{s}-\varepsilon_{\infty}\right) \omega \tau}{1+\omega^{2} \tau^{2}}$ e $\varepsilon_{s}$ e $\varepsilon_{\infty}$ são, respectivamente, os limites de $\tilde{\varepsilon}$ quando $\omega \rightarrow 0 \mathrm{e}$ $\omega \rightarrow \infty$, isto é, $\chi_{1}=\varepsilon_{\infty}-1$ e $\chi_{2}=\varepsilon_{s}-\varepsilon_{\infty}$. As duas últimas equações, para $\varepsilon^{\prime}(\omega)$ e $\varepsilon^{\prime \prime}(\omega)$ são conhecidas como equações de Debye [2] e foram deduzidas por Debye a partir de hipótese moleculares.

$\mathrm{O}$ vetor deslocamento elétrico $D(t)$ consiste de duas componentes: $\varepsilon^{\prime} E(t)$ em fase com o campo elétrico e $\varepsilon^{\prime \prime} E(t)$ em quadratura com o campo elétrico e atrasada.

Uma das aproximações feitas na física, às vezes inconscientemente, é aquela da simultaneidade. Usualmente assume-se que o valor de uma função desconhecida e suas derivadas que ocorrem numa equação diferencial são todas avaliadas no mesmo instante. Por exemplo: na modelagem do decaimento radioativo supõe-se que $\frac{d N(t)}{d t}=-\lambda N(t)$ ou na modelagem da cinética química $\frac{d C(t)}{d t}=-k C(t)$. Existem inúmeros exemplos em física e biologia nos quais a velocidade de variação de uma função do tempo depende do valor passado desta função [4]. No caso de dielétricos certamente os mecanismos de orientação molecular não ocorrem simultaneamente com o campo e sim com um certo atraso ou defasagem. Para levar em conta este fato, vamos postular um novo modelo, escrevendo a Eq. (1) sob a forma:

$$
\tau \frac{d P_{2}(t)}{d t}=\chi_{2} \varepsilon_{0} E(t)-P_{2}(t-r)
$$

onde $r$ é uma medida do atraso da resposta dos dipolos em relação ao campo elétrico aplicado. A equação diferencial (6) é conhecida como [4,5,6] "Delay Differential Equation".

Na hipótese do campo elétrico ser dado por $E(t)=$ $E_{0} u(t)$ obtém-se o seguinte para a Eq. (6):

$$
\begin{gathered}
P_{2}(t)=\chi_{2} \varepsilon_{0} E_{0}\left[1+\frac{1+\alpha \tau}{\tau(\beta-\alpha)} \exp (\beta t)-\right. \\
\left.-\frac{1+\beta \tau}{\tau(\beta-\alpha)} \exp (\alpha t)\right] .
\end{gathered}
$$

sendo $\alpha$ e $\beta$ as raízes reais não nulas da equação $s \tau+\exp (-r s)=0$. Usou-se como condição de contorno $[6,7] \quad P_{2}(t)=0$ para $t \in[-r, 0]$.

A equação diferencial (6) também pode ser resolvida numericamente (ver apêndice) ou em forma de série utilizando a transformada de Laplace [5]. Neste último caso a solução encontrada é:

$$
P_{2}(t)=\chi_{2} \varepsilon_{0} E_{0} \sum_{n=0}^{\infty} \frac{(-1)^{n+2}(t-n r)^{n+1}}{\tau^{n+1}(n+1) !} u(t-n r)
$$

Os três resultados da integração da equação diferencial (6) conduzem a resultados numéricos idênticos.

Analogamente para campos do tipo $E(t)=$ $E_{0} \exp (i \omega t)$ obtém-se a solução da equação diferencial (6), no domínio da frequiência, usando a técnica da transformada de Fourier [8], obtendo-se a expressão:

$$
P_{2}(\omega)=\frac{\chi_{2} \varepsilon_{0} E(\omega)}{\cos (\omega r)+i[\omega \tau-\sin (\omega r)]} .
$$

Para campos alternados senoidais de frequiência $\omega$ tem-se $E(\omega)=E_{0}$. Nesta nova condição a permissividade relativa verdadeira e o fator de perdas serão dados, respectivamente pelas equações:

$$
\varepsilon^{\prime}=\varepsilon_{\infty}+\frac{\left(\varepsilon_{s}-\varepsilon_{\infty}\right) \cos (\omega r)}{[\cos (\omega r)]^{2}+[\omega \tau-\sin (\omega r)]^{2}}
$$




$$
\varepsilon^{\prime \prime}=\frac{\left(\varepsilon_{s}-\varepsilon_{\infty}\right)[\omega \tau-\sin (\omega r)]}{[\cos (\omega r)]^{2}+[\omega \tau-\sin (\omega r)]^{2}} .
$$

\section{Cálculo numérico}

Para exemplificar numericamente o novo modelo acima exposto, vamos considerar um cristal de $\mathrm{KBr}+\mathrm{Ca}^{++}$com as seguintes características: $\varepsilon_{\infty}=$ $2,33, \varepsilon_{s}-\varepsilon_{\infty}=2,45, \tau=0,001 s$.

Fixando o valor de $r=0,0003 \mathrm{~s}$, obtém-se para as raízes reais da equação $s \tau+\exp (-r s)=0$ os valores: $\alpha=-1631,34$ e $\beta=-5937,79$. A Fig. 1 mostra $P_{2}(t)$ em função do tempo segundo as Eqs. (2) e (7) Observa-se uma acentuada a diferença entre os dois modelos, em particular entre o inicio e a saturação. A medida que $\frac{r}{\tau}<0,0001$ a diferença entre os dois modelos desaparece completamente.

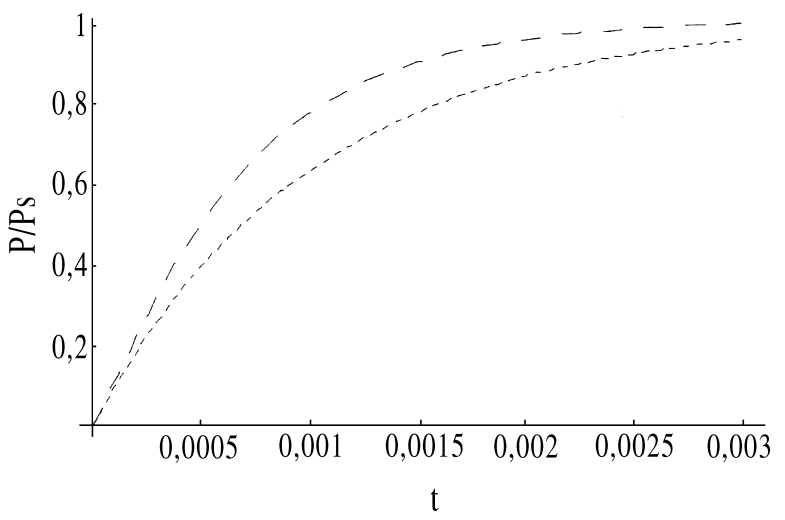

Figura 1 - A Eq. (2) está representada pela linha de traço fino.A linha de traço largo representa qualquer das Eqs. (7), (8) ou (A6) para $r=0,0003$. O tempo é medido em segundo.

Para campos elétricos senoidais de freqüência $\omega$, as Figs. 2 e 3 mostram os valores de $\varepsilon^{\prime}(\omega)$ e $\varepsilon^{\prime \prime}(\omega)$ no qual utilizamos $r=0,0003$. Para valores pequenos da relação $\frac{r}{\tau}<0,0001$ a diferença entre os dois modelos é imperceptível.

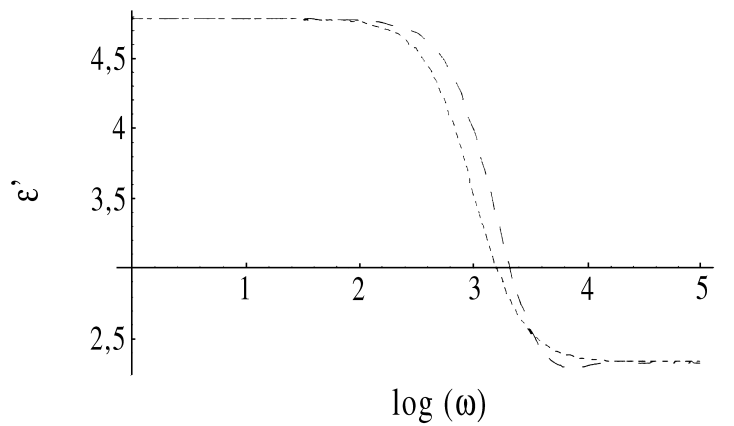

Figura 2 - Permissividade relativa $\varepsilon^{\prime}$ segundo o modelo clássico (traço fino) e de acordo com a Eq. 10 para o modelo modificado (traço largo), onde adotou-se o valor $r=0,0003$.

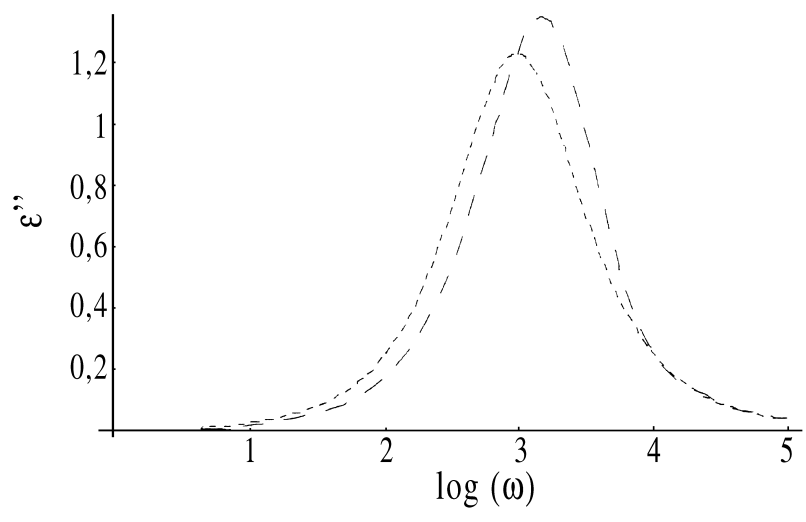

Figura 3 - Fator de perda $\varepsilon^{\prime \prime}$ segundo o modelo clássico (linha de traço fino) e de acordo com a Eq. (11) para o modelo modificado ( linha de traço largo), onde adotou-se o valor $r=0,0003$.

A Fig. 4 mostra o diagrama de Cole-Cole para $r=0,0003$. De maneira similar, quando $\frac{r}{\tau}<0,0001$, o diagrama de Cole-Cole não apresenta diferença entre os dois modelos.

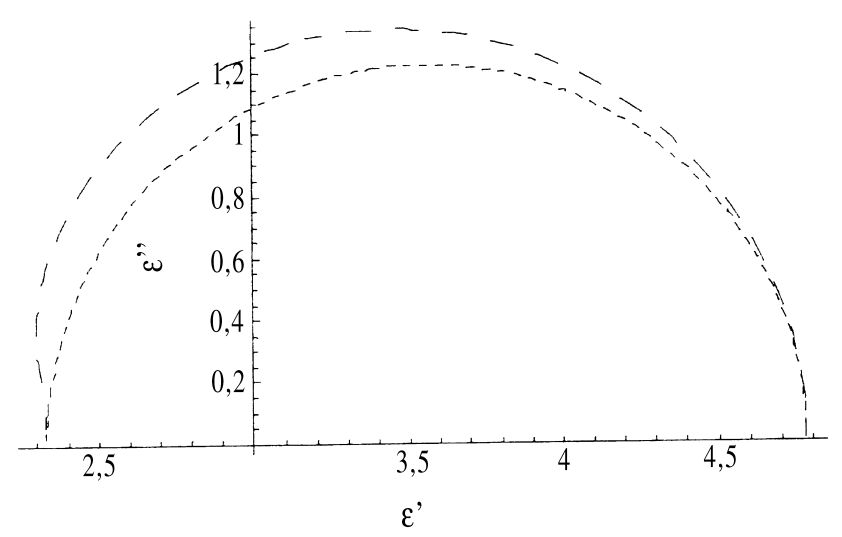

Figura 4 - Diagrama de Cole-Cole para o modelo clássico (linha de traço fino) e para o modelo modificado (linha de traço largo), onde adotou-se o valor $r=0,0003$.

\section{Conclusão}

O problema aqui exposto não é comumente tratado na literatura. Conforme pode-se constatar para valores pequenos da relação $r / \tau$ a diferença entre os dois modelos é desprezível, o mesmo não ocorrendo para valores de $r$ da ordem de grandeza de $\tau$, não importando se o problema lida com campos elétricos constantes ou alternados. O problema aqui resolvido serve como exemplo para o estudo de sistemas similares, tais como, em farmacologia, a absorção de drogas pelo organismo humano. 


\section{Referências}

[1] R. Fournier, Les Isolants en Électrotechnique, Concepts et théorie (Editions Eyrolles, Paris, 1986), p. 8, 65.

[2] W.F. Brown, Dielektrika Handbuch Der Physik (Springer-Verlag, Berlin, 1956), p. 119.

[3] V.V. Daniel, Dielectric Relaxation (Academic Press, London, 1967), p. 12-19.

[4] R.D. Driver, Ordinary and Delay Differential Equation (Springer-Verlag, N.Y., 1977), p. 225.

[5] V. Ditkine et A. Proudnikov, Calcul Opérationnel (Editions Mir, Moscou, 1979), p. 246.

[6] R.D. Driver, Journal of Mathematical Analysis and Applications 157, 591 (1991).

[7] D. Zwillinger, Handbook of Differential Equations (Academic Press, San Diego, 1998), p. 230-237.

[8] P.H. Hsu, Analisis de Fourier (Addison Wesley Iberoamericana, USA, 1987), p. 74, 83, 87.

\section{Apêndice}

Seja a equação diferencial:

$$
\tau \frac{d P_{2}(t)}{d t}=\chi_{2} \varepsilon_{0} E_{0}-P_{2}(t-r)
$$

onde se supõe que $\frac{r}{\tau} \quad<1$. Baseado no "Method of Steps" proposto por Driver [4], façamos a seguinte transformação:

$$
P_{2}(t)=y(t)-\chi_{2} \varepsilon_{0} E_{0}
$$

Onde $y(t)$ é uma função a ser determinada. A equação diferencial (A1) pode ser escrita como:

$$
\tau \frac{d y(t)}{d t}=-y(t-r) \text {. }
$$

Se $r$ for pequeno e definirmos o passo no tempo como sendo $r$, a derivada na Eq. (A3) aproximada por:

$$
\frac{d y(t)}{d t} \approx \frac{y(j r)-y(j r-r)}{r} \quad j=1,2,3 \ldots
$$

Consequientemente, a Eq. (A3) transforma-se na relação de recorrência:

$$
y(j r)=\left(1-\frac{r}{\tau}\right)^{j} y(0)
$$

Voltando à Eq. (A2) e lembrando que $y(0)=$ $\chi_{2} \varepsilon_{0} E_{0}$ ficamos com:

$$
P_{2}(j r)=\chi_{2} \varepsilon_{0} E_{0}\left[1-\left(1-\frac{r}{\tau}\right)^{j}\right] j=
$$
$1,2,3, \ldots$

(A6) 